Mit einem Zitat von Ulrich Beck leitet Robert Feustel den Schluss seiner Ausführungen ein: „Es ist keine Schande zu bekennen, dass auch uns Sozialwissenschaftlern die Sprache versagt, angesichts der Wirklichkeit, die uns überrollt.“ (Beck 2014, so zit in: Feustel 2019: 144) Im Kontext der Forschung zum aktuellen Aufstieg der Rechten stößt, so Feustel, „die methodisch seriöse Wissenschaft an ihre Grenzen“, weshalb mit Rechten reden „nur bedingt [hilft]“ (ebd.: 144), die aktuell relevanten gesellschaftlichen Transformationsprozesse zu verstehen. Während ich viele der Darlegungen teile, überkommt mich bei der Lektüre immer wieder ein Unbehagen. Ich teile die warnenden Worte zum Umgang mit den neuen Medien, den Verweis auf die schwierige Rolle, in der sich Wissenschaft befindet, wenn sie „als ideologisch voreingenommen verunglimpft" (ebd.: 141) wird und sehe auch den tiefen Graben zwischen den Welten der neurechten Milieus und den (kritischen) Sozialwissenschaften. Jedoch, wenn uns als Wissenschaftler_innen angesichts all dessen die Sprache versagt, dann sagt es mehr über unsere eigene Hilflosigkeit aus, mit dem Wandel der Gesellschaft umzugehen, als über die Konstitution von Gesellschaft selbst.

Im Kern lautet meine Antwort, die ich ausgehend von Rancières PolitikVerständnis zu geben versuche: Gesellschaftliche Ordnungen waren schon immer zerklüftet, multipel und umkämpft. Der Aufstieg der Rechten führt dies aktuell deutlich vor Augen. Für uns als Wissenschaftler_innen ist das unangenehm, da wir direkt erleben, dass unsere Normvorstellungen, ja das System Wissenschaft selbst, angegriffen werden und an Gewicht verlieren. Wir werden, ob gewollt oder nicht, aus unseren Komfortzonen gerissen. Ich plädiere für einen unaufgeregten Zugang zu den aufbrechenden Konfliktlinien und einen dezidiert politischen Zugriff auf diese Fragen, was einen differenzierten Umgang mit der Frage,mit Rechten reden?' impliziert.

Für Rancière (2002: 12) bedeutet Politik die Ausdehnung des Dissensraums. Ihr liegt ein Unvernehmen zugrunde, womit ein „Typus von Sprechsituation" benannt wird, in welchem beide Seiten des Dialoges sich zwar vernehmen, sich gegenseitig aber nicht verstehen. Beide Seiten sagen „Weiß“, verstehen darunter aber etwas Unterschiedliches (ebd.: 9f.). Politik ist der 
Kampf zwischen „zwei Wahrnehmungswelten“ (Rancière 2014: 148), die aufeinandertreffen und einen Streit über die Deutungshoheit eingehen (ebd.: 236). In einer solchen Praxis wird, so Slavoj Žižek (2014: 16), der Rahmen, „durch den wir die Welt wahrnehmen und uns in ihr bewegen“, selbst verhandelt. Dabei wird nicht nur Zukunft verändert, sondern Zeit insgesamt affiziert: Wenn der Rahmen, durch den wir Welt betrachten, ausgetauscht wird, „dann erschafft dieses Neue sich rückwirkend seine eigene Möglichkeit, seine eigenen Ursachen/Bedingungen“"(ebd.: 112). Hintergrund dieses Arguments ist die postfundamentalistische These, dass nichts sein muss wie es ist und jede gesellschaftliche Formation Produkt von politischer Praxis ist. Jede soziale Ordnung lässt sich begründen und aus jeder lassen sich Wirkmächte ableiten, jedoch kann sie nicht vollumfänglich objektiviert werden - faktisch ist Ordnung also nie. Dies ist kein Plädoyer für Beliebigkeit. Einmal etablierte Ordnung wirkt, schafft Strukturen, setzt Normen, bedeutet Herrschaft und ein spezifisches Set an Machtbeziehungen. Sie beruht in letzter Instanz aber immer ,nur' auf der Behauptung, dass es alleine diese und keine andere Ordnung geben kann, weshalb eine jede Ordnung verändert werden kann (Marchart 2010).

Was heißt dies nun für die hier relevante Frage, ob, und wenn ja, wie , mit Rechten reden?‘. Mir scheinen drei Punkte von Relevanz:

Erstens: Der Aufstieg der Rechten und deren Diskursstrategien verdeutlichen keineswegs eine gänzlich neue Situation für die Sozialwissenschaften. Sie markieren aber unverkennbar einen neuen Pol in Auseinandersetzungen um Gesellschaft (Mullis 2017). Polarisierung wurde in den letzten Jahrzehnten fast ausschließlich als Konflikt zwischen emanzipatorischen sozialen Bewegungen und der kapitalistischen Vergesellschaftung beschrieben. Viele kritische Sozialforscher_innen, mich eingeschlossen, haben sich an die Seite von sozialen Bewegungen gestellt und deren emanzipatorische Potentiale betont. Viele erkannten in der globalen Protestwelle des Jahres 2011 und den anhaltenden Mobilisierungen der folgenden Jahre einen progressiven Aufbruch. Der Aufstieg der Rechten in Deutschland (Decker/Brähler 2018; Heitmeyer 2018) und insbesondere das Erstarken der AfD kamen überraschend. Brachen sie doch mit dem Glauben, dass sich rechts der CDU/CSU keine Partei werde etablieren können. Für uns als Wissenschaftler_innen bedeutet dies, dass wir selbst über die Zuschreibung als Teil des Establishments Objekt von Anfeindung werden; dass die von uns hochgehaltene Wahrheit - auch deren poststrukturalistische Wendung - offensiv in Frage gestellt wird; dass wir in der Forschung zu und über die Rechten uns nicht mehr auf dem Standpunkt des ,Solidarischseins mit' ausruhen können - wir also mit unserer eigenen Positionalität weit stärker hadern. All dies bedeutet den Auszug aus der Komfortzone und, ob wir wollen oder nicht, das Eintreten in politische Auseinandersetzungen. Sprachlosigkeit ist ein schlechter Begleiter und eher Effekt der eigenen Verunsicherung in einer Welt im Wandel, und nicht Produkt der Unfähigkeit, den Wandel empirisch zu erfassen.

Zweitens gilt es den von Feustel im Anschluss an John Law noch defensiv formulierten Punkt, dass Realität nicht definitiv und singulär ist, unbedingt zu unterstreichen. Gesellschaft ist ein pluraler Prozess, der von Herrschaft und Machtbeziehungen geordnet und von Kämpfen durchzogen ist. Sozialwissenschaften sahen sich immer schon mit der Herausforderung 
konfrontiert, eine Welt zu beschreiben, die nicht in Gänze fassbar ist. Um sich dieser Welt anzunähern, wurden gerade in der qualitativen Sozialforschung sensible Verfahren entwickelt, die sich allzu starrer Kategorisierung entziehen und dennoch erlauben, dominante Muster von Vergesellschaftung zu beschreiben. Der Forschung entspringen widerstreitende Positionen, was aber keine Schwäche ist. So bedeutet Wissenschaft immer auch Streit um Deutung und nicht per se die Produktion von Wahrheit. Oder: Die Wahrheit ist gerade im begründeten Streit zu finden. Womöglich war die Umkämpftheit von Gesellschaft durch den Dunst postdemokratischer Vernebelung in den 1990ern und 200oern gut kaschiert, latent war der Kampf aber immer.

Wenn nun Feustel, weiter Law folgend, argumentiert, dass „gegenwärtige sozialwissenschaftliche Methoden an der Beschreibung dieser chaotischen Normalität hoffnungslos [scheitern]“ (Feustel 2019: 142), dann kann dies nur zweierlei heißen: Entweder sie scheiterten angesichts des niemals abwesenden Chaos schon immer oder sie sind heute noch genauso angemessen wie vorher. Womöglich stimmt beides, aber mit Sicherheit sollte empirische Sozialforschung nicht eingestellt werden. Auch die Größen der Frankfurter Schule Horkheimer, Fromm, Marcuse und Adorno entwickelten ihre Theorien nicht ohne Empirie; und es waren gerade Fromms empirische Studien zum autoritären Charakter in den 1930er Jahren, die den Mitgliedern des Instituts für Sozialforschung die große Gefahr des herannahenden Faschismus aufzeigten.

Heute wird diese Tradition von den Autor_innen der „Mittestudien“ um Oliver Decker - seit 2018 „Autoritarismus-Studie“ - (Decker/Brähler 2018) sowie der „Deutschen Zustände“ um Wilhelm Heitmeyer (2018) fortgeführt - Arbeiten, die ich auf keinen Fall missen möchte. Zustimmen möchte ich Feustel hingegen, dass angesichts der bisweilen unübersichtlichen quantitativen Kategorisierungsflut „dichte Beschreibungen und theoretische Deutungen [...] vielleicht mehr [verraten]“ (ebd.: 144) und gestärkt werden müssen. Denn solche Forschung erlaubt, sich an die multiplen und bisweilen ambivalenten Mechanismen von Vergesellschaftung aus verschiedenen Richtungen anzunähern. Sie erlaubt Prozesse der Subjektivierung, Krisenerfahrungen und gesellschaftliche Deutungen in Relation zu theoretischen Überlegungen zu setzen, ohne im Prozess des Forschens sowie in der Darstellung der Ergebnisse allzu stark kategorisieren zu müssen. Kurzum, sie erlaubt Ambivalenz, und dies ist unerlässlich, sollen politische Praxen und das Zustandekommen von Einstellungen verstanden werden. Empirische - und nicht zuletzt auch qualitative - Arbeiten zur Rechten sind also notwendig. Ein Rückzug in Theoriearbeit wäre fatal, weil dies auch den Rückzug aus dem Dialog mit Gesellschaft bedeuten würde: Wir werden in aller Regel nicht aufgrund von Theoriearbeit für Interviews, Gespräche mit der Zivilgesellschaft oder die Teilnahme an öffentlichen Podien angefragt, sondern wegen empirischer Forschung.

Drittens möchte ich für einen differenzierten Umgang mit der Frage ,mit Rechten reden?' plädieren. Dies bedeutet, an mancher Stelle sind Gespräche angebracht, andernorts sind sie strikt zu verweigern. Zentral ist für mich aber, zwischen verschiedenen Typen des Gesprächs zu unterstreichen, die Feustel leider immer wieder vermengt, wenn er das öffentliche Reden auf Podien im gleichen Satz mit Forschungspraxis zusammen nennt. 
Strikt zu verweigern ist das Gespräch mit Kadern der extremen Rechten im öffentlichen Raum. Dies keineswegs aus moralischen Gründen, sondern aufgrund einer politischen Einschätzung. So ist es ein zentrales Mittel der aktuell erstarkenden Rechten, politische Auseinandersetzung auf das Terrain von Kultur, Medien, Kunst und Wissenschaft zu verlagern. Ihr Ziel ist, den "Gegner aus einer Defensivposition heraus mit dessen eigenen Mitteln“ (Weiß 2017: 54) zu schlagen. Es geht den Rechten darum, den öffentlichen Diskurs sukzessive nach rechts zu verschieben. Zentrales Mittel hierfür ist die Selbstinszenierung als Opfer, als von Eliten, Medien und Wissenschaft entrechtete Minderheit, die einen legitimen Kampf um Anerkennung führt - dass das Ergebnis dieses Kampfes die Auflösung der demokratischen Ordnung und die Abschaffung der Minderheitenrechte selbst ist, wird geflissentlich unterschlagen. Die Gesprächsverweigerung kann diese Mechanik durchbrechen. Denn wenn ein jeder Dialog, unabhängig von Gesprächsverlauf und Inhalt, nur auf die Inszenierung als Opfer hinausläuft, ist das Gespräch an sich das Problem. Das Gegenargument, dass die Einbindung in den Dialog den rechten Spuk zu entzaubern vermöge, erweist sich in der konkreten Erfahrung, etwa mit der SVP in der Schweiz, der FPÖ in Österreich und immer deutlicher auch mit der AfD, als Irrweg und hilft den Parteien, sich zu etablieren und deren von Fremdenhass, Nationalismus und Antifeminismus durchzogenen Positionen zu normalisieren. Von diesem Standpunkt aus ist notwendig auch in der Wissenschaft - gerade im Rahmen qualitativer Forschung - das Gespräch mit Kadern der extremen Rechten und deren Vordenker_innen strikt zu verweigern. Ihnen gehört keine Plattform geboten, zumal sie die normative Grundlage derselben verachten. Um deren Positionen nachzuvollziehen, reicht es ihre Schriften zu lesen.

Notwendig ist aber das Gespräch mit jenen Menschen zu suchen, die sich im Dunstkreis dieser Bewegungen verorten oder ,schlicht' rechtsautoritäre Parteien wählen. Ziel solcher Gespräche, gerade wenn sie im Forschungskontext stattfinden, sollte es, das scheint mir wichtig, keinesfalls sein, die Narrative und Deutungen lediglich als solche zu erfassen. Dies würde nur deren Multiplikation und Verbreiterung dienen. Allgemein reicht Dekonstruktion als kritische Praxis der Intervention nicht mehr aus. Denn wenn unterschiedliche Rahmen, durch die die Welt gesehen wird, in Konkurrenz gesetzt werden, und die wissenschaftlichen Paradigmen der Untersuchung nicht zu dem Rahmen gehören, durch den die Gesprächspartner_innen blicken, dann wird das Ergebnis der Untersuchung nicht vermittelbar sein.

Was wir heute als ,Fake News‘ erkennen, so ärgerlich und wissenschaftlich falsch sie im Einzelnen auch sein mögen, sollte keinesfalls einfach als Irrweg, falsches Bewusstsein oder Unvernunft abgetan werden. Hätte es den Begriff schon am Vorabend der Französischen Revolution gegeben, hätte ihn Louis XVI. wohl auch gegen die republikanischen Bestrebungen ins Feld geführt. Gleiches gilt für Charles de Gaulle oder Kurt Kiesinger, als sie sich mit der Revolte der 68er konfrontiert sahen. Ich möchte keine Gleichsetzung der politischen Projekte nahelegen, sehr wohl aber verdeutlichen, dass jede politische Auseinandersetzung bisweilen auf Kontra-Faktischem und anmaßenden Behauptungen basiert. Wir müssen anerkennen, dass solche Diskurspraxen Versuche sind, Ordnung zu errichten und Welt neu 
zu gestalten. Das empörte Beharren auf den Rahmen der Faktizität und Wahrheit selbst wird jene, die sich davon lösen, nicht wieder ins Bild holen. Wir kommen nicht umhin, in politische Auseinandersetzungen einzutreten: Demokratie, die Universalität der Menschenrechte, soziale Gerechtigkeit und eine offene und plurale Gesellschaft verteidigen wir nicht altruistisch in ihrem eigenen Namen, sozusagen um ihretwillen, sondern weil sie das Ringen um Fakten und Wahrheit überhaupt erst ermöglichen und damit Freiheit zu streiten stiften. Eine Freiheit, die die rechten Demagogen zu unterbinden suchen. Es ist Aufgabe von Wissenschaftler_innen, diese Freiheit, die das System Wissenschaft selbst ausmacht, aber auch konstitutiv für eine demokratische Gesellschaft ist, zu verteidigen.

Ziel von Forschung müsste es also sein, den demokratischen Rahmen zu stärken und eine gemeinsame Grundlage des Austauschs zu schaffen. Das Gespräch mit Rechten sollte darauf zielen, die Subtexte und die sozialpsychologischen Wirkmechanismen zu verstehen, die das Kontra-Faktische so anziehend machen. Arlie Russel Hochschilds Plädoyer aus Fremd in ihrem Land (2017), hierfür die „Empathiemauer zu überwinden“(ebd.: 20), reicht, so lesenswert ihr Buch auch ist, nicht aus, und kann bisweilen problematisch werden. Sie hilft zu verstehen, wieso sich weiße Männer in den Südstaaten der USA an den Rand gedrängt fühlen. Was ihr abgeht, ist die Kehrseite dieser Konstruktion zu benennen: Den Hass und die Gewalt, die aus dem Glauben an die Ungleichwertigkeit der Menschen und die Überlegenheit der ,weißen Rasse“ hervorgehen. Am Ende der Lektüre bleibt ein Gefühl des Mitleids und dies ist fatal. So wichtig auf der einen Seite empirische Forschung und eine partielles ,Reden mit' also sind, so wichtig ist auf der anderen Seite die fundierte Einbettung der Gespräche in eine umfassende Gesellschaftsanalyse, denn Welt ist immer mehr als das je konkrete Narrativ. Empathie darf Widerspruch nicht verhindern, Grenzen des Demokratischen gilt es deutlich zu markieren.

\section{Autor innen}

Daniel Mullis ist Humangeograph. Seine Interessen liegen in der politischen Philosophie, Sozialprotesten sowie Krisendynamiken, aktuell insbesondere im Erstarken regressiver Politiken.

mullis@hsfk.de

\section{Literatur}

Decker, Oliver / Brähler, Elmar (Hg.) (2018): Flucht ins Autoritäre. Rechtsextreme Dynamiken in der Mitte der Gesellschaft. Gießen: Psychosozial-Verlag.

Feustel, Robert (2019): Substanz und Supplement. Mit Rechten reden, zu Rechten forschen? Eine Einladung zum Widerspruch. In: $\mathrm{s}$ u b $\backslash \mathrm{u} \mathrm{r}$ b a n. zeitschrift für kritische stadtforschung, 7(1/2), 137-145.

Heitmeyer, Wilhelm (2018): Autoritäre Versuchungen. Berlin: Suhrkamp.

Hochschild, Arlie Russell (2017): Fremd in ihrem Land. Eine Reise ins Herz der amerikanischen Rechten. Frankfurt: Campus.

Marchart, Oliver (2010): Die politische Differenz. Berlin: Suhrkamp.

Mullis, Daniel (2017): Das Ende der Postdemokratie, den Pessimismus überwinden. In: Prokla 47/3, 487-494. 
Rancière, Jacques (2002 [1995]): Das Unvernehmen. Politik und Philosophie. Frankfurt am Main: Suhrkamp.

Rancière, Jacques (2014): Erfindung des Möglichen. Interviews 2006-2009. Wien: Passagen.

Weiß, Volker (2017): Die autoritäre Revolte. Die Neue Rechte und der Untergang des Abendlandes. Stuttgart: Klett-Cotta.

Žižek, Slavoj (2014): Was ist ein Ereignis. Frankfurt am Main: Fischer. 